\title{
Do immune checkpoint inhibitors increase sensitivity to salvage chemotherapy?
}

\author{
Khalil Saleh¹, Nadine Khalifeh-Saleh¹, Hampig Raphaël Kourie*,1, Fadi Nasr ${ }^{1}$ \& Georges \\ Chahine ${ }^{1}$ \\ ${ }^{1}$ Hematology-Oncology Department, Faculty of medicine, Saint Joseph University, Beirut, Lebanon \\ * Author for correspondence: hampig.kourie@hotmail.com
}

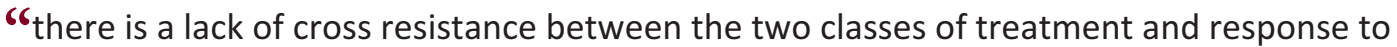
chemotherapy is maintained independently of previous exposure to $\mathrm{ICl}$. This retrospective study also showed that $\mathrm{ICl}$ exposure could enhance tumor sensitivity to conventional chemotherapy."
\end{abstract}

First draft submitted: 27 October 2017; Accepted for publication: 19 December 2017; Published online: 26 January 2018

Keywords: checkpoint inhibitors • salvage chemotherapy • sensitivity

Immune checkpoint inhibitors (ICI) are profoundly changing the treatment of solid and hematologic malignancies and becoming an integral part of the management of cancer patients. These agents led to distinguished durable response in approximately $20 \%$ of patients when given a second or subsequent line of therapy with a manageable safety profile [1]. We thought that prior treatment with chemotherapy can enhance tumor sensitivity to PD$1 / \mathrm{PD}-\mathrm{L} 1$ inhibitors. It has been reported that chemotherapeutic agents could induce immune-reactive effects such as cross-presentation of tumor antigens or MHC class 1 molecules and reducing T regulatory cells or myeloid derived suppressor cells which increases the accumulation of helper T cell at the tumor site [2-4]. Recently, Peng et al. showed that chemotherapy enhances PD-L1 overexpression via the NF-kb and creates an immunosuppressive tumor microenvironment in ovarian cancer [5]. In fact, the high expression of PD-L1 and the decreased T regulatory cells or myeloid derived suppressor cells was associated with better response to ICI [6]. However, do ICIs increase sensitivity to salvage chemotherapy?

\section{What is the rationale?}

Some reports in the literature showed that response to chemotherapy can be increased in patients exposed to vaccine-based immunotherapy in different types of cancer. For example, Antonia et al. reported an objective clinical response of $61.9 \%$ to chemotherapy after vaccination with dendritic cells transduced with the full-length wildtype p53 gene in patients with advanced stage small-cell lung cancer [7]. The treatment with a cytochrome P450 $1 \mathrm{~B} 1$ vaccine was associated with high response to salvage chemotherapy in patients who developed immunity to CYP1B1 [8]. In hormone refractory prostate cancer, docetaxel showed a significantly longer time to progression in patients immunized with recombinant viral vectors containing the PSA and B7.1 genes compared with patients who received docetaxel alone in a randomized Phase II study [9]. Similarly, Wheeler et al. reported that patients who received chemotherapy after an autologous tumor-pulsed dendritic cell vaccine exhibited significantly longer time to tumor recurrence and survival advantage than patients treated with chemotherapy alone in patients with glioblastoma [10]. These reports suggest that immunotherapy could sensitize tumor cells and enhance the effect of chemotherapy.

\section{What about immune checkpoint?}

Concerning ICI, it has been demonstrated that inhibition of PD- 1 on $\mathrm{CD}^{+}{ }^{+}$-tumor-infiltrating lymphocytes using anti-PD-1 can restore cytokine secretion and T cell proliferation [11] rising the hypothesis that immune checkpoint inhibition can activate the immune system, change the natural history of the disease and the microenvironment rendering tumor cells more sensitive to chemotherapy. 
Recently, Leger $e$ al. presented in abstract form a response rate of $27 \%$ to salvage chemotherapy in patients with advanced non-small-cell lung cancer (NSCLC) who had progressed on checkpoint inhibitor. The odds ratio for achieving partial response was 0.30 . Docetaxel was the most frequently administered chemotherapy in these patients (62\%) [12]. Furthermore, Schvartsman et al. reported the objective response rate (ORR) to singleagent chemotherapy after exposure to PD-1 or PD-L1 inhibitors in patients with advanced NSCLC at MD Anderson Cancer Center. In his cohort of 28 patients, the confirmed ORR to single-agent chemotherapy was 29\%, with another three patients who presented unconfirmed partial responses raising the ORR to $39 \%$. The median progression-free survival was 4.7 months and the overall survival was 9.0 months. Docetaxel was the chemotherapy most frequently used after progression on ICI. However, all patients in Schvartsman's cohort did not respond to immunotherapy (ORR: 0\%) and four patients had EGFR-mutation known to increase sensitivity to conventional chemotherapy [13]. It seems that the ORR to salvage chemotherapy is higher than response reported with secondline docetaxel in advanced NSCLC. Overall there are no data showing that patients having durable response on immunotherapy will have the same or a more favorable response rate than patients with rapidly progressive disease after ICI. The question remains: do patients who rapidly progress after beginning of immunotherapy have more response to conventional chemotherapy?

\section{Does sequencing of chemotherapy \& ICI maximize the outcome in cancer patients?}

Emerging data showed an excellent response rate to chemotherapy after exposure to first-line ICI in patients with metastatic urothelial carcinoma (64\%) [14]. Szabados et al. compared the response rate to salvage chemotherapy following ICI exposure of two cohorts of patients (total 28 patients): the first cohort concerned patients who received ICI in first-line setting followed by chemotherapy and the second one consisted on patients who received chemotherapy followed by ICI then chemotherapy after progression. Information concerning ICI in this study was not given since patients were included in clinical trials. The response rate in the first cohort was $64 \%$ comparable to the $50 \%$ of response to standard platinum-based chemotherapy in the first-line setting [15]. The response rate to salvage chemotherapy in the second cohort was $21 \%$ in third line which is concordant with the results in patients who progressed on first line without prior exposure to ICI [16]. The authors concluded that there is a lack of cross resistance between the two classes of treatment and response to chemotherapy is maintained independently of previous exposure to ICI. This retrospective study also showed that ICI exposure could enhance tumor sensitivity to conventional chemotherapy.

\section{Future perspective}

All the above showed that previous exposure to PD-1/PD-L1 inhibitors could increase sensitivity to salvage chemotherapy in patients who progressed on ICI. However, this is based on retrospective findings reported on small populations and in much selected groups of patients. More investigations should be done to confirm if patients who initially responded to immunotherapy had also an important response rate to salvage chemotherapy and if a specific sequencing of standard chemotherapy and PD-1/PD-L1 inhibitors could improve patients' survival. Ongoing randomized trials may validate these findings.

Financial \& competing interests disclosure

The authors have no relevant affiliations or financial involvement with any organization or entity with a financial interest in or financial conflict with the subject matter or materials discussed in the manuscript. This includes employment, consultancies, honoraria, stock ownership or options, expert testimony, grants or patents received or pending, or royalties.

No writing assistance was utilized in the production of this manuscript.

\section{References}

1 Saleh K, Khalife-Saleh N, Haddad EE, Kourie HR. Negative predictive biomarkers of checkpoint inhibitors in hyper-progressive tumors. Biomark. Med. 11(10), 819-821(2017).

2 Wan S, Pestka S, Jubin RG, Lyu YL, Tsai Y-C, Liu LF. Chemotherapeutics and radiation stimulate MHC class I expression through elevated interferon-beta signaling in breast cancer cells. PloS One 7(3), e32542 (2012).

3 Ohtsukasa S, Okabe S, Yamashita H, Iwai T, Sugihara K. Increased expression of CEA and MHC class I in colorectal cancer cell lines exposed to chemotherapy drugs. J. Cancer Res. Clin. Oncol. 129(12), 719-726 (2003).

4 Suzuki E, Kapoor V, Jassar AS, Kaiser LR, Albelda SM. Gemcitabine selectively eliminates splenic Gr-1+/CD11b+ myeloid suppressor cells in tumor-bearing animals and enhances antitumor immune activity. Clin. Cancer Res. Off. J. Am. Assoc. Cancer Res. 11(18), 6713-6721 (2005). 
5 Peng J, Hamanishi J, Matsumura N et al. Chemotherapy induces programmed cell death-ligand 1 overexpression via the nuclear factor- $\mathrm{kB}$ to foster an immunosuppressive tumor microenvironment in ovarian cancer. Cancer Res. 75(23), 5034-5045 (2015).

6 Remon J, Chaput N, Planchard D. Predictive biomarkers for programmed death-1/programmed death ligand immune checkpoint inhibitors in nonsmall cell lung cancer. Curr. Opin. Oncol. 28(2), 122-129 (2016).

7 Antonia SJ, Mirza N, Fricke I et al. Combination of p53 cancer vaccine with chemotherapy in patients with extensive stage small cell lung cancer. Clin. Cancer Res. Off. J. Am. Assoc. Cancer Res. 12(3 Pt 1), 878-887 (2006).

8 Gribben JG, Ryan DP, Boyajian R et al. Unexpected association between induction of immunity to the universal tumor antigen CYP1B1 and response to next therapy. Clin. Cancer Res. Off. J. Am. Assoc. Cancer Res. 11(12), 4430-4436 (2005).

9 Arlen PM, Gulley JL, Parker C et al. A randomized Phase II study of concurrent docetaxel plus vaccine versus vaccine alone in metastatic androgen-independent prostate cancer. Clin. Cancer Res. Off. J. Am. Assoc. Cancer Res. 12(4), 1260-1269 (2006).

10 Wheeler CJ, Das A, Liu G, Yu JS, Black KL. Clinical responsiveness of glioblastoma multiforme to chemotherapy after vaccination. Clin. Cancer Res. Off. J. Am. Assoc. Cancer Res. 10(16), 5316-5326 (2004).

$11 \mathrm{He} \mathrm{J,} \mathrm{Hu} \mathrm{Y,} \mathrm{Hu} \mathrm{M,} \mathrm{Li} \mathrm{B.} \mathrm{Development} \mathrm{of} \mathrm{PD-1/PD-L1} \mathrm{pathway} \mathrm{in} \mathrm{tumor} \mathrm{immune} \mathrm{microenvironment} \mathrm{and} \mathrm{treatment} \mathrm{for} \mathrm{non-small} \mathrm{cell}$ lung cancer. Sci. Rep. [Internet]. 5 (2015). www.ncbi.nlm.nih.gov/pmc/articles/PMC4538573

12 Response to salvage chemotherapy following exposure to immune checkpoint inhibitors in patients with non-small cell lung cancer. $J$. Clin. Oncol. 35(15) Suppl. [Internet]. http://ascopubs.org/doi/abs/10.1200/JCO.2017.35.15_suppl.9084

13 Response rates to single-agent chemotherapy after exposure to immune checkpoint inhibitors in advanced non-small cell lung cancer ScienceDirect [Internet]. www.sciencedirect.com/science/article/pii/S0169500217304336

14 Szabados B, van Dijk N, Tang YZ et al. Response rate to chemotherapy after immune checkpoint inhibition in metastatic urothelial cancer. Eur. Urol. doi:10.1016/j.eururo.2017.08.022. (2017) (Epub ahead of print).

15 von der Maase H, Sengelov L, Roberts JT et al. Long-term survival results of a randomized trial comparing gemcitabine plus cisplatin, with methotrexate, vinblastine, doxorubicin, plus cisplatin in patients with bladder cancer. J. Clin. Oncol. Off. J. Am. Soc. Clin. Oncol. 23(21), 4602-4608 (2005).

16 Sonpavde G, Sternberg CN, Rosenberg JE, Hahn NM, Galsky MD, Vogelzang NJ. Second-line systemic therapy and emerging drugs for metastatic transitional-cell carcinoma of the urothelium. Lancet Oncol. 11(9), 861-870 (2010). 
